\title{
Collapse load analysis for circumferentially cracked cylinder subjected to combined torsion and bending moments
}

\author{
Sutham Arun $^{1 *}$ and Thongchai Fongsamootr ${ }^{2}$ \\ ${ }^{1}$ School of Engineering, University of Phayao, Phayao, Thailand \\ ${ }^{2}$ Department of Mechanical Engineering, Chaing Mai University, Chaing Mai, Thailand
}

\begin{abstract}
Cylinder is one of the most commonly used components which has a risk of having circumferential cracks, especially in the welding zone. When cracks are discovered, it is necessary to perform the failure strength assessment of cracked cylinder and the limit load play an important part as the input of the assessment. At present, the limit load solution for circumferential cracked cylinder under combined bending and torsion can be estimated by using the methods of equivalent moment or biaxial failure parameter. However, these methods still have some limitations. The main aim of this paper is to propose the alternative method for predicting the failure moment of circumferential cracked cylinder under combined bending and torsion. The method used in this paper is based on the modification of biaxial failure parameter and the data from finite element analysis. Details of this method is presented in this paper.
\end{abstract}

\section{Introduction}

Cylinders used in power station, petroleum, steel and other industries may experience internal pressure, axial load, bending and torsion. This makes cylinder at the risk of having circumferential cracks, especially in the welding zone. When cracks are discovered, it is necessary to perform the failure strength assessment to prevent the severe accidents.

Plastic limit load is one of the most important factors when performing structurally integrity assessment of cracked structure. According to this, many analytical and finite element (FE) solutions have been developed for predicting the plastic limit load of circumferential cracked cylinder [1-4]. However, these solutions are usually developed for Mode I type loading (opening mode) of internal pressure, axial tension and bending. The effects of Mode III type of torsion moment are not included in the solutions.

As cylinder may be subjected to torsion during installation or piping arrangement, ignoring the effect of torsion when deriving the limit load solution may results in incomplete or overestimated solutions. At present, several methods for predicting the failure load of cracked cylinder under combined torsion and bending moments have been developed. These methods are based on the equivalent collapse moment and biaxial failure parameter $[5,6]$. However, these methods still have some limitations when they were used to estimate the failure load.

The main aim of this paper is to provide the alternative method to predict the failure load for circumferential cracked cylinder under combined bending and torsion. The method is based on the modification of biaxial failure parameter and the data from finite element analysis.

\section{Research background}

\subsection{Description of geometry}

Figure 1 shows the geometry of circumferential cracked cylinder considered in this work. The dimension of the cylinder is described by its mean radius $R_{m}$ and wall-thickness $t$. The crack is assumed to be symmetric with respect to the $y$-axis. The total angle of crack and the crack depth measured from cylinder inner surface are defined by $2 \theta$ and $a$. The cylinder is subjected to combined external bending moment $M_{B}$ about $x$-axis and external torsion moment $M_{T}$ about $z$-axis.
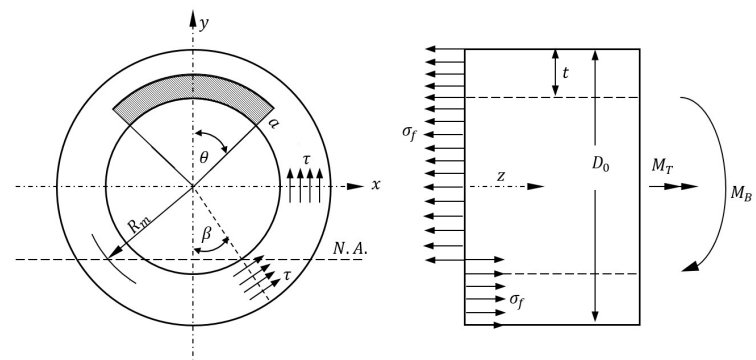

Fig. 1. Nomenclature and stress distribution of a cylinder with a circumferential crack.

According to the geometry defined in Figure 1, the plastic collapse moment for cylinder without crack under pure bending and pure torsion conditions can be given by;

$$
\begin{aligned}
& M_{B 0}=4 R_{m}^{2} t \sigma_{f} \\
& M_{T 0}=\frac{2}{\sqrt{3}} \pi R_{m}^{2} t \sigma_{f}
\end{aligned}
$$

\footnotetext{
* Corresponding author: sutham.arun@gmail.com
} 
where $M_{B 0}$ and $M_{T 0}$ are the plastic collapse moment for uncracked cylinder subjected to bending and torsion, respectively. Eqs. (1) and (2) are developed based on an elastic-perfectly plastic material with flow stress $\sigma_{f}$.

\subsection{Limit load solutions for cylinders with circumferential crack}

For circumferential cracked cylinder under pure bending, the solution of collapse bending moment is provided in ASME B\&PV Code Section XI [7]. This solution was developed based on net-section-collapse (NSC) principle which can be expressed as follows :

$$
\begin{aligned}
& \beta=\frac{1}{2}\left(\pi-\frac{a}{t} \theta\right) \\
& M_{B F}=2 \sigma_{f} R_{m}^{2} t\left[2 \sin (\beta)-\frac{a}{t} \sin (\theta)\right]
\end{aligned}
$$

where $\sigma_{f}$ is the flow stress, $\beta$ is the neutral angle of the cylinder cross section, see Figure 1. Eqs. (3) and (4) are applicable to the case which whole crack is inside the tensile stress zone $(\theta+\beta \leq \pi)$.

The collapse moment solution for circumferential cracked cylinder under pure torsion has been mentioned in few literatures. This solution is derived based on the NSC approach. For the case of constant-depth crack, the solution is given as follows [6];

$$
M_{T F}=\frac{2}{\sqrt{3}} \pi R_{m}^{2} t \sigma_{f}\left(1-\frac{\theta}{\pi} \frac{a}{t}\right)
$$

However, there are some evidences showing that Eq.(5) can provide accurate prediction only for the case of short cylinder length or shallow surface crack $[6,8]$.

For the case of cracked cylinder subjected to combined bending and torsion moments, Bezensek et al. [5] showed that the collapse moment can be described as equivalent moments $M_{e q}$ defined by;

$$
M_{e q}=\sqrt{M_{B}^{2}+M_{T}^{2}}
$$

where $M_{B}$ and $M_{T}$ are the external bending moment and the external torsion moment at collapse. Bezensek [5] also found that when the torsion stress $\tau$ acting on the crack section was less than $20 \%$ of flow stress $\left(\tau / \sigma_{f}<0.2\right)$, the values of $M_{e q}$ were almost constant. This implies that $M_{e q}$ can be used to predict the collapse plastic failure for the case of small amount of torsion.

As $M_{e q}$ is not valid for the case of large torsion moment, Li et al. [6] defined a non-dimensional failure parameter $r$ which can be used to estimate the collapse failure of cylinder for general magnitude of torsion moment. This parameter is given by

$$
r=\sqrt{\left(\frac{M_{B}}{M_{B F}}\right)^{2}+\left(\frac{M_{T}}{M_{T F}}\right)^{2}}
$$

where $M_{B F}$ and $M_{T F}$ are the plastic collapse moments of cracked cylinder subjected to pure bending moment and pure torsion moment, respectively. $\mathrm{Li} \mathrm{[6]} \mathrm{also} \mathrm{found} \mathrm{that}$ for any crack depth and crack angle, the value of $r$ is almost equal to 1 . However, there is some difficulties in using Eq.(7) in the prediction of failure moment. This is because there is no standard solution for computing the value of $M_{T F}$ for general cases. Therefore, FE analysis may be the only way to obtain the value of $M_{T F}$.

\section{Finite element analysis model}

The FE models of cylinder with circumferential crack were generated in ABAQUS [9]. These models are used to analyze the correctness of the biaxial failure parameter defined in Eq.(7). Details of the models are given in the following sections.

\subsection{Model construction}

Figures 2 and 3 present the three-dimensional FE model of straight cylinder with circumferential crack. In all cases, the outer diameter of a cylinder, $D_{0}$ is $600 \mathrm{~mm}$, and the length of the cylinder is eight times larger than the outer diameter in order to avoid boundary effects on the crack ligament $[6,10]$. To prevent problem associated with shear locking, 20-node quadratic brick element with reduced integration $(\mathrm{C} 3 \mathrm{D} 20 \mathrm{R})$ is used to generate the models.

The material used for cylinder is a high-ductility stainless steel $[6,10]$. The Young's modulus is selected as $200 \mathrm{GPa}$ with Poisson's ratio of 0.3 . The stress-strain relationship is assumed to be elastic-perfectly plastic with the yield stress of $372 \mathrm{MPa}$.

The FE analysis contains 2 loading steps. The first step is to apply the specified value of torsion moment on the cylinder end. After the torsion moment is maintained at the given value, the bending moment is then applied to the cylinder in the second loading step. This process is performed until the maximum moment is achieved.

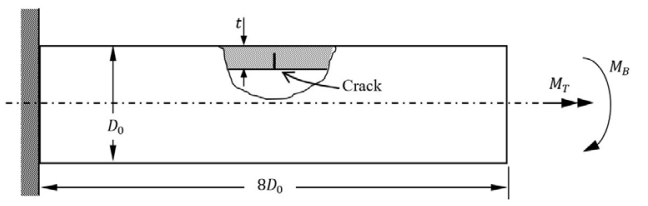

Fig. 2. Model of a cracked cylinder under bending moment and torsion moment.

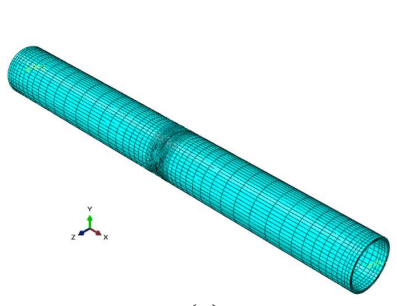

(a)

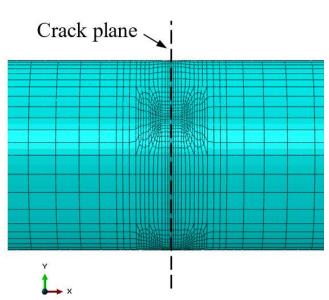

(b)
Fig. 3. (a) The specimen mesh overview and (b) mesh near the crack in longitudinal direction. 


\subsection{Limit load analysis}

The models are analyzed using elastic-plastic analysis with large deformation formulation. The further post-processing is performed to obtain the value of limit load. For the case of cylinder subjected to a single load, e.g. pure bending or pure torsion, the collapse moment is defined using the twice-elastic-slope (TES) method [11]. For the case of cylinder subjected to combined bending and torsion moments, the plastic collapse load is defined from the value of the maximum bending moment which the model can carry. The reason in choosing this method is given in the next section.

\subsection{Validation of the model}

The confidence in the FE analysis is gained by comparing the current FE-results with the results from Hasegawa et al. [10]. Figure 4 shows that the present FE results agree very well with Hasegawa's work, with the difference less than $5 \%$.

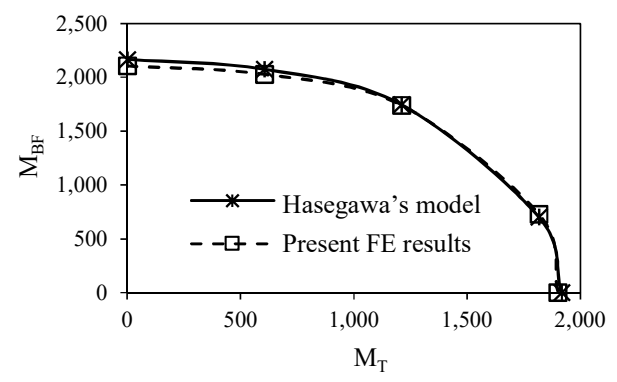

Fig. 4. Comparison of plastic collapse bending moment for cylinder with crack of $2 \theta=90$ and $a / t=1.0$.

\section{Results and discussion}

\subsection{Global behaviour}

Figure 5 shows the relationship between the normalized bending moment $\left(M / M_{B 0}\right)$ and bending angle $\left(\theta_{b}\right)$ as a function of normalized torsion moment $M_{T} / M_{T 0}$. This is the load-displacement curve for the case of $R_{m} / t=10, a / t=0.75$ and $2 \theta=90^{\circ}$. The figure shows that in elastic region, the slope of loaddisplacement curve is almost equal for any value of $M_{T} / M_{T 0}$. This implies that when material behaves elastically, the elastic beam theory can still be used to predict the global behavior of the circumferential cracked cylinder subjected to combined torsion and bending moment.

From Figure 5, it can be observed that the bending moment decreases gradually with increasing of $\theta_{b}$ after the maximum bending moment is achieved. According to this, TES method may not be the proper method for estimating the collapse moment. Therefore, for the case of combined moments, the plastic collapse bending moment is defined from the value of the maximum bending moment which the model can carry.

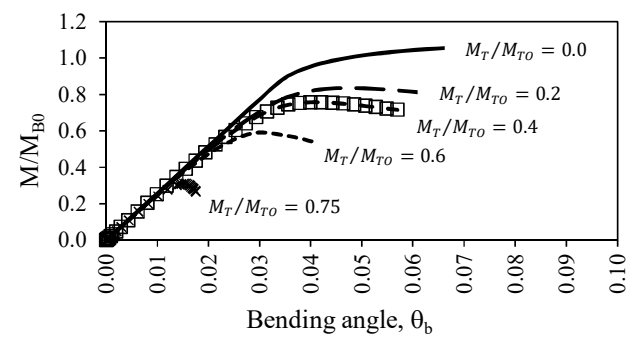

Fig. 5. Load - displacement curve for cylinder with crack of $2 \theta=90$ and $a / t=0.75$.

\subsection{Equivalent collapse moment $\left(M_{e q}\right)$}

The collapse bending moment and collapse torsion moment obtained from $\mathrm{FE}$ analysis are used to compute the values of $M_{e q}$ by Eq.(6). Figure 6 illustrates that the values of $M_{e q}$ is almost constant when the value of $M_{T} / M_{T 0}$ are less than 0.25 . This information corresponds to the works presented in literatures [5] which indicates that $M_{e q}$ can be used to predict the plastic collapse failure for the case of small amount of torsion.

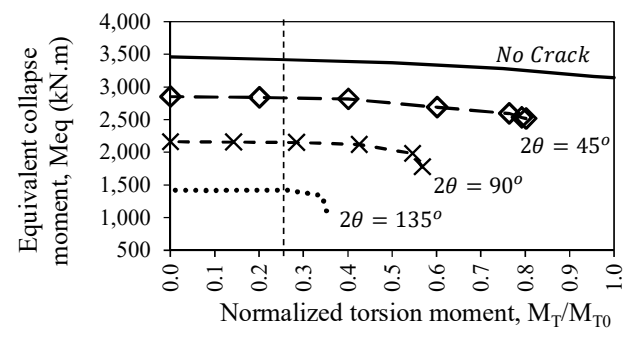

Fig. 6. Relationship between equivalent collapse moment and the collapse torsion moment for the case of $R_{m} / t=10$ and $a / t=0.75$.

\subsection{Biaxial failure parameter $(r)$}

Figure 7 illustrates the relationship between $M_{B} / M_{B F}$ and $M_{T} / M_{T F}$ for the case of $R_{m} / t=10$. As presented in Eq.(7), this relationship is in the form of equation of circle centered at the origin with radius $r$. Figure 7 shows that the values of $r$ is almost equal to 1 for any crack depth and crack angle. This result confirms that the parameter $r$ can be used to predict the plastic collapse moment for a general range of torsion moment.

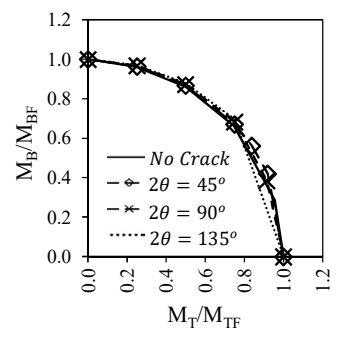

(a)

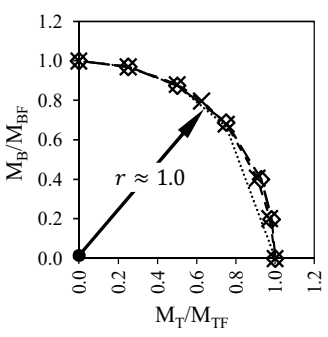

(b)
Fig. 7. Relationship between $M_{B} / M_{B F}$ and $M_{T} / M_{T F}$ : (a) $a / t=0.25$ and (b) $a / t=0.75$. 


\subsection{Modified biaxial failure parameter $\left(r_{0}\right)$}

As mentioned before, it is necessary to assess the values of $M_{T F}$ when Eq.(7) is employed to estimate the failure moment. The only way to obtain this value is from FE analysis. To overcome this issue, Eq.(7) is modified by replacing $M_{B F}$ and $M_{T F}$ with $M_{B 0}$ and $M_{T 0}$ as follows;

$$
r_{0}=\sqrt{\left(\frac{M_{B}}{M_{B 0}}\right)^{2}+\left(\frac{M_{T}}{M_{T 0}}\right)^{2}}
$$

Since $M_{B 0}$ and $M_{T 0}$ can be computed by Eqs.(1) and (2), the problem of computing $M_{T F}$ is solved. Figure 8 presents the relationship between $M_{B} / M_{B 0}$ and $M_{T} / M_{T 0}$. It can be seen that the relationship is still in the form of circle equation but the radius $r_{0}$ decreases with the increase of cracked area. In other words, $r_{0}$ is a function of $a / t$ and $\theta / \pi$. From this, the following quadratic approximation of $r_{0}$ is proposed based on the data from the FE analysis;

$$
\begin{aligned}
r_{0}= & 0.985+0.4\left(\frac{a}{t}\right)-0.04\left(\frac{\theta}{\pi}\right)-1.97\left(\frac{a}{t}\right)\left(\frac{\theta}{\pi}\right) \\
& -0.33\left(\frac{a}{t}\right)^{2}+0.84\left(\frac{\theta}{\pi}\right)^{2}
\end{aligned}
$$

Eq.(9) provides the values of $r_{0}$ within $9 \%$ of FE results at crack angle $2 \theta \leq 135^{\circ}$ and $5 \leq R_{m} / t \leq 20$.

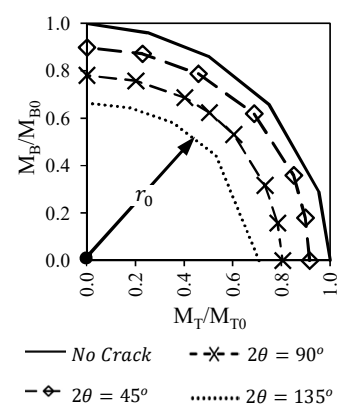

(a)

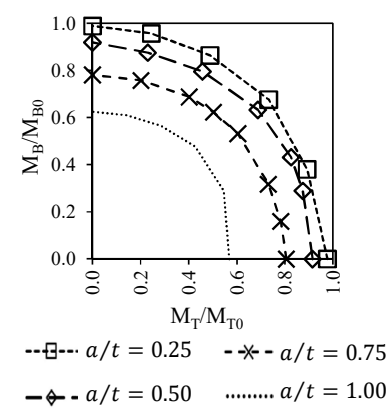

(b)
Fig. 8. Relationship between $M_{B} / M_{B 0}$ and $M_{T} / M_{T 0}$ for the case of (a) $a / t=0.75$ and (b) $2 \theta=90^{\circ}$

\section{Conclusion}

In this work, the 3D FE model is used to analyze plastic collapse load for circumferential cracked cylinder subjected to combined bending and torsion moments. The analysis is based on the methods of equilibrium collapse moment $M_{e q}$ and biaxial failure parameter $r$. The following conclusions can be made :

1. As material behaves elastically, the global behavior of cracked cylinder can be predicted by the elastic beam theory.

2. The equivalent moment $M_{e q}$ can be employed to predict the plastic collapse moment for the case of small amount of torsion, $M_{T} / M_{T 0} \leq 0.25$.

3. The biaxial failure parameter $r$ can be used to estimate the collapse moment of cylinder for general magnitude of torsion moment. However, it may not be convenient in using this parameter, since there is no standard solution of $M_{T F}$ for general cases.

4. In order to avoid the calculation of $M_{T F}$, the parameter $r$ is modified to be $r_{0}$ as shown in Eq.(8).

5. The values of $r_{0}$ can be estimated by Eq.(9) for the case of $2 \theta \leq 135^{\circ}$ and $5 \leq R_{m} / t \leq 20$, with the difference less than $9 \%$ from the FE analysis.

\section{References}

1. S.Rahman, "Net-section-collapse analysis of circumferentially cracked cylinders Part II: idealized cracks and closed-form solutions, "Engineering Fracture Mechanics, vol.61, pp.213-230, 1998.

2. Y.J.Kim, D.J.Shim, N.Kamran, Y.J.Kim, S.S.Hwang and J.S.Kim, "Finite element based plastic limit loads for cylinders with part-through surface cracks under combined loading," International Journal of Pressure Vessels and Piping, vol.80, pp.527-540, 2003.

3. Y.Lei and P.J.Budden, "Limit load solutions for thinwalled cylinders with circumferential cracks under combined internal pressure, axial tension and bending," The Journal of Strain Analysis for Engineering Design, vol.39 (6), pp.673-683, 2004.

4. Y.Lei, Y.Li and Z.Gao, "Global limit load solutions for thick-walled cylinders with circumferential cracks under combined internal pressure, axial force and bending moment Part I: Theoretical solutions," International Journal of Pressure Vessels and Piping, vol.114-115, pp.23-40, 2014.

5. B.Bezensek, Y.Li, K.Hasegawa and P.H.Hoang, "Inclusion of torsion loads in Section XI flaw evaluation procedures for pipes containing circumferential planar surface crack-like flaw on the basis of Limit Load Analysis," Journal of Pressure Vessel Technology, vol.134(3), 2012.

6. Y.Li, K.Hasegawa, P.H.Hoang and B.Bezensek, "Prediction Method for Plastic Collapse of Pipes Subjected to Combined Bending and Torsion Moments," in Proceedings of ASME 2010 pressure vessel \& piping conference, Bellevue, Washington, USA, July 18-22, 2010.

7. ASME Boiler \& Pressure Vessel Code, Section XI. Rules for inservice inspection of nuclear power plant components, American Society of Mechanical Engineers, New York; 2010.

8. H.W.Ryu, J.J.Han and Y.J.Kim, "Limit loads for circumferentially cracked pipes under combined cending and torsion with tension," in Proceedings of ASME 2013 pressure vessel \& piping conference, Paris, France, July 14-18, 2013.

9. Hibbitt, Karlsson and Sorensen Inc, "ABAQUS/Standard User Manual," Rhode Island.

10. K.Hasegawa Y.Li and K.Osakabe, "Collapse loads for circumferentially through-wall cracked pipes subjected to combined torsion and bending moments," Engineering Fracture Mechanics, vol.123, pp.77-85, 2014.

11. M.Muscat D.Mackenzie and R.Hamilton, "A work criterion for plastic collapse," International Journal of Pressure Vessels and Piping, vol.80(1), 2003. 